\title{
Quantitative Review Techniques of Edge Detection Operators.
}

\author{
Yogesh Uttam Patil, Dr. S. D. Patil, Dr.Abdul Jabbar Shaikh Azad \\ (Department of Computer Science, PSGVP Mandals Arts, Science \& Commerce Sr.College Shahada (M.H.) \\ (Department of Computer Science, Arts, Commerc \& Science College Navapur (M.H.). \\ (Department of Computer Science, PSGVP Mandals Arts, Science \& Commerce Sr.College Shahada (M.H.).
}

\begin{abstract}
An edge is a boundary between an object and its background. If the edges in an image can be identified accurately, all of the objects can be located and basic properties such as area, perimeter, and shape can be measured. Since computer vision involves the identification and classification of objects in an image. So for this purpose edge detection is an important tool. This technique helps to localize the significant variants of the graylevel images and to identify the physical phenomena. This information is very useful in image registration, compression, enhancement and restoration, reconstruction, understanding, recognition.
\end{abstract}

Keywords: Canny , Prewit, Roberts, Sobel Edge Detection operator.

\section{INTRODUCTION}

1. Image Analysis

2. Segmentation

4. Results

5. Application
3. Edge Detection

6. Conclusion

\section{IMAGE ANALYSIS}

The basic structure of an Image is an array of matrix.

- Each unit element of a matrix is a pixel in the displayed image.

- The quality of an image is directly proportional to the number of pixels in the image.

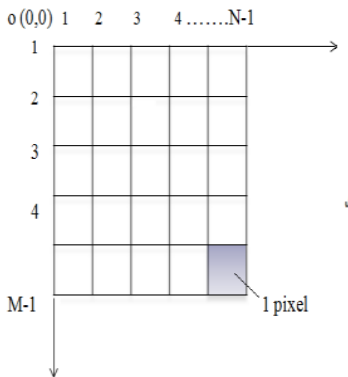

\section{SEgMENTATION}

This means sub-division of an image into its constituent regions or objects.

It is based on one of two basic properties of intensity values:

i) Discontinuity: Abrupt changes in intensity.

Example: Edges (Line, Point, Edge detections are in this category)

ii) Similarity: Regions similar according to some predefined criteria

\section{EDGE DETECTION}

If edges are identified accurately from an image, all of the objects can be located. We can calculate
i) Area
ii) Perimeter
iii)Shape

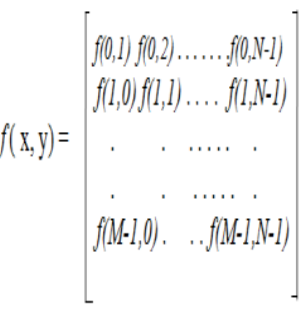

Edge Detection Techniques are basically of two types:

a) Gradient,

b) Laplacian.

Gradient: It uses first order derivative

$$
\nabla f=\left[\begin{array}{l}
\frac{\partial f}{\partial x} \\
\frac{\partial f}{\partial y}
\end{array}\right]
$$

Magnitude of this vector

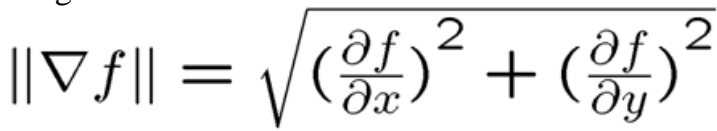

Laplacian: It uses second order derivative 


$$
\nabla^{2} f=\frac{\partial^{2} f}{\partial x^{2}}+\frac{\partial^{2} f}{\partial y^{2}}
$$

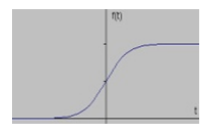

Signal

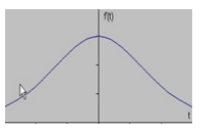

Gradient

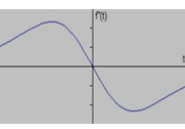

Laplacian

\section{Gradient Based Operators}

Sobel Operator: It consists of a pair of $3 \times 3$ convolution mask

\begin{tabular}{|l|r|r|}
\hline-1 & 0 & +1 \\
\hline-2 & 0 & +1 \\
\hline-1 & 0 & +1 \\
\hline \multicolumn{4}{|c|}{$\frac{\partial f}{\partial x}$}
\end{tabular}

\begin{tabular}{|c|c|c|}
\hline+1 & +2 & +1 \\
\hline 0 & 0 & 0 \\
\hline-1 & -2 & -1 \\
\hline
\end{tabular}

$$
\begin{aligned}
& \frac{\partial f}{\partial x}=\left(Z_{7}+2 Z_{8}+Z_{9}\right)-\left(Z_{1}+2 Z_{2}+Z_{3}\right) \\
& \frac{\partial f}{\partial y}=\left(Z_{3}+2 Z_{6}+Z_{9}\right)-\left(Z_{1}+2 Z_{4}+Z_{7}\right)
\end{aligned}
$$

Robert's cross operator: It is simple, quick to compute, 2D spatial gradient measurement of an image. It consists of $2 \times 2$ convolution mask.

\begin{tabular}{|c|c|}
\hline-1 & 0 \\
\hline 0 & 1 \\
\hline
\end{tabular}

\begin{tabular}{|c|c|}
\hline 0 & -1 \\
\hline 1 & 0 \\
\hline
\end{tabular}

$$
\begin{aligned}
& \frac{\partial f}{\partial x}=\left(Z_{9}-Z_{5}\right) \\
& \frac{\partial f}{\partial y}=\left(Z_{8}-Z_{6}\right)
\end{aligned}
$$

Here we can see one kernel is simply the other rotated by $90^{\circ}$.

\begin{tabular}{|c|c|c|}
\hline-1 & -1 & -1 \\
\hline 0 & 0 & 0 \\
\hline 1 & 1 & 1 \\
\hline
\end{tabular}

Prewitt's operator: It is similar to Sobel operator and is used for detecting vertical and horizontal edges in images.

\begin{tabular}{|l|l|l|}
\hline-1 & 0 & 1 \\
\hline-1 & 0 & 1 \\
\hline-1 & 0 & 1 \\
\hline
\end{tabular}

$$
\begin{aligned}
& \frac{\partial f}{\partial x}=\left(Z_{7}+Z_{8}+Z_{9}\right)-\left(Z_{1}+Z_{2}+Z_{3}\right) \\
& \frac{\partial f}{\partial y}=\left(Z_{3}+Z_{6}+Z_{9}\right)-\left(Z_{1}+Z_{4}+Z_{7}\right)
\end{aligned}
$$

\section{Laplacian Based Operator}

Canny's Edge Detection Algorithm (Optimal edge detector)

The algorithm steps are as follows

1. Convolve the image with a two dimensional Gaussian filter to smooth it.

2. Differentiate the image in two orthogonal directions.

3. Calculate the gradient amplitude and direction.
4. Relate the edge direction to a direction that can be traced.

5. Perform non-maximal suppression. Any gradient value that is not a local peak is set to zero. The gradient direction is used in this process.

6. Threshold these edges to eliminate 'insignificant' edges. 


\section{PERFORMANCE OF DIFFERENT Comparison using intensity values} EDGE DETECTION ALGORITHM

1.Rice

\begin{tabular}{|l|l|}
\hline Operator & Root Mean Square Error \\
\hline Sobel & 0.5121 \\
\hline Prewitt & 0.5174 \\
\hline Roberts & 0.5182 \\
\hline Canny & $\mathbf{0 . 5 0 2 5}$ \\
\hline
\end{tabular}

2. Pears

\begin{tabular}{|l|l|}
\hline Operator & Root Mean Square Error \\
\hline Sobel & 0.8368 \\
\hline Prewitt & 0.8368 \\
\hline Roberts & 0.8297 \\
\hline Canny & $\mathbf{0 . 8 3 5 1}$ \\
\hline
\end{tabular}

3. Coins

\begin{tabular}{|l|l|}
\hline Operator & Root Mean Square Error \\
\hline Sobel & 0.5481 \\
\hline Prewitt & 0.5491 \\
\hline Roberts & 0.5491 \\
\hline Canny & $\mathbf{0 . 5 4 7 2}$ \\
\hline
\end{tabular}

\section{GRAPHICAL INTERPRETATION OF THE RESULTS}
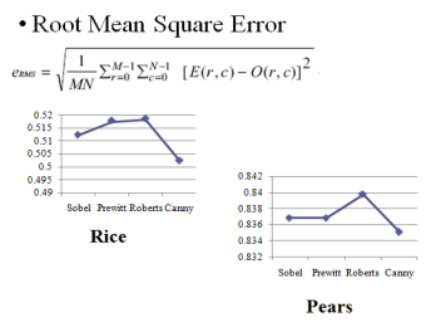

\section{APPLICATIONS}

- Used to detect Thumb impressions

- Analyze the direction of flow of a river.

- Identify a particular object, its area, shape etc.

- Used in medical sector.

- Identify leakage or cracks in microchips.

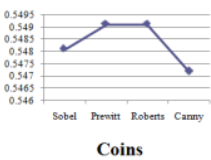

\section{CONCLUSION}

Edge detection techniques are most important factor to identify images in computer vision.This technique helps to localize the significant variants of the gray-level images and to identify the physical phenomena. We are confirmed that out of all the Canny's operator is best to locate the intensity of the edge of an image.

\section{REFERENCES}

[1] Gonzalez R. C. and Woods R. E. Digital Image Processing, 2nd ed., Prentice Hall. 2005.

[2] Roushdy M., Comparative Study of edge detection algorithms applying on the

grayscale noisy image using morphological filter, ICGST International Journal on Graphics, Vision and Image Processing,

[3] Dr.S.Vijayarani , Mrs.M.Vinupriya, Performance Analysis of Canny and Sobel Edge Detection Algorithms in Image Mining

[4] Tzu-Heng Henry Lee, Edge Detection Analysis

[5] Mitra basu, senior member, ieee -Gaussian-based edge-detection methods-A surveyll, ieee transactions on systems, man, and cybernetics-part c: applications and reviews, vol. 32, no. 3, august 2002.

\section{Books:}

[6] Digital Image Processing, 2nd Ed., Gonzalez \& Woods, Pearson Education.

[7] Fundamentals of Image processing, A.K. Jain, Prentice Hall of India Publication, 1995 